\title{
Synthetic Approach for Novel Fluorine Substituted $\alpha$-Aminophosphonic Acids Containing 1,2,4-Triazin-5-One Moiety as Antioxidant Agents
}

\author{
Mohammed S. T. Makki, Reda M. Abdel-Rahman, Abdulrahman S. Alharbi \\ Department of Chemistry, Faculty of Science, King Abdul Aziz University, Jeddah, KSA \\ Email: *aalharbi2017@hotmail.com
}

How to cite this paper: Makki, M.S.T., Abdel-Rahman, R.M. and Alharbi, A.S. (2018) Synthetic Approach for Novel Fluorine Substituted $\alpha$-Aminophosphonic Acids Containing 1,2,4-Triazin-5-One Moiety as Antioxidant Agents. International Journal of Organic Chemistry, 8, 1-15.

https://doi.org/10.4236/ijoc.2018.81001

Received: December 8, 2017

Accepted: January 13, 2018

Published: January 16, 2018

Copyright $\odot 2018$ by authors and Scientific Research Publishing Inc. This work is licensed under the Creative Commons Attribution International License (CC BY 4.0).

http://creativecommons.org/licenses/by/4.0/

\begin{abstract}
Novel fluorine substituted $\alpha$-amino phosphonic acids containing 1,2,4-triazin5 -one (6a-f) have been obtained from fluoroacylation of 6-(2'-amino-5'-nitrophenyl)-3-thioxo-1,2,4-triazin-5(4H)-one (1) followed by ammonilysis to give the corresponding 3 -amino-derivative 3 . Condensation of compound 3 with nitro/halogenated aromatic aldehydes yielded the Schiff bases 4 . The simple addition of diethyl phosphonate to compound 4 produced the $\alpha$-amino phosphonates $\mathbf{5}$. Acidic hydrolysis of compound $\mathbf{5}$ produced the fluorine substituted $\alpha$-amino acids derivatives 6 . Structures of the new compounds have been established with the help of elemental analysis and spectral measurements. Also, the products evaluated as antioxidants, where the fluorinated $\alpha$-amino phosphonic acids 6 are more active than the other synthesized systems.
\end{abstract}

\section{Keywords}

Synthetic, Fluorine $\alpha$-Amino Acids, 1,2,4-Triazin-5-One Moiety, Antioxidants Activity

\section{Introduction}

Recently, $\alpha$-amino phosphonic acids and $\alpha$-amino phosphonates have a vital importance of research chemists [1] [2] [3], which is due to these family of compounds display, enzymatic inhibitors for HIV protease antagonists [4] and collagenase inhibitors [5]. Also, they use as anticancer [6], antibacterial [7], antiviral [8] and antioxidant [9] agents. On the other hands, functionally 1,2,4-triazines have unique properties for biological, medicinal and pharmacological chemistry 
[10] [11] [12]. Phosphorus compounds bearing and/or containing 1,2,4-triazine moieties exhibit a significant attention due to the specific biological properties [13] [14] [15] [16] [17]. Also, the introduction of fluorine atoms to heterocyclic nitrogen systems, mostly improve their physical, chemical and medical properties [18] [19] [20] [21]. In the present work, we focused on the reactivity of functional 1,2,4-triazines towards different reagents followed by simple addition of diethyl phosphonate to obtain a novel fluorine substituted $\alpha$-amino phosphonic acids which considered as $\alpha$-amino acids analog, in view of antioxidant activity.

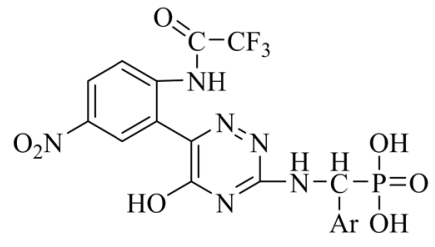

$\alpha$-amino phosphonic acids

\section{Chemistry}

The phosphorylation of amino-organic heterocyclic systems often improves their biological activity because of the P-O bond stores energy for metabolic processes [1]. Also, the chemistry of N-phosphoryl heterocyclic indicates that these compounds form dimensional polymeric chain via intermolecular $\mathrm{P}^{-} \mathrm{O}^{-} \ldots \ldots .{ }^{+} \mathrm{NH}$ hydrogen bond [2]. Moreover, the reactivity of the dipolar ion structures of the tautomeric form of $\alpha$-amino phosphonates is due to the higher electron-withdrawing properties of two phenoxy and $\mathrm{P}=\mathrm{O}$ groups. Thus, the $\alpha$-amino-phosphonate group has a high degree of stability against any reagent attack [3]. To deduce the aims of this work, 6-(2'-Amino-5'-nitrophenyl)3-thioxo-1,2,4-triazin- $5(2 \mathrm{H}, 4 \mathrm{H})$-one $(1)$ as a starting material obtained from reflux of 5-nitroisatin with thiosemicarbazide in aq. $\mathrm{NaOH}$ (Scheme 1).

Fluoroacylation of compound 1 by boiling with ethyl 2,2,2-trifluoroacetate in THF yielded [22] 2,2,2-trifluoro-N-[2-(5-hydroxy-3-thioxo-2,3-dihydro-1,2,4triazin-6-yl)-4-nitrophenyl] acetamide (2), which upon ammonilysis by reflux with liquid ammonia in ethanol produced [23] 3-amino-6-[2'-(trifluoroacetamido-5"'-nitrophenyl)]-1,2,4-triazin-5(4H)-one (3) (Scheme 2). Condensation of compound 3 with various nitro and halogenated aromatic aldehydes in boiling ethanol yielded the corresponding Schiff bases 4 (Scheme 2).

The main aims of the present work produce a novel fluorine substituted $\alpha$-amino acids containing 1,2,4-triazinone moiety. The addition of compounds with phosphorus hydrogen bonds to azomethine $(\mathrm{HC}=\mathrm{N}-\mathrm{Ar})$ bonds provides an economical method for the synthesis of organophosphorus derivatives. Thus, the addition of diethyl phosphonate to Schiff bases by warm at $80^{\circ} \mathrm{C}-100^{\circ} \mathrm{C}$ along $6 \mathrm{~h}$ with a few drops of triethylamine produced [24] $\alpha$-amino phosphonates 5 which upon acid hydrolysis afforded [25] the novel fluorinated $\alpha$-amino phosphonic acids 6 as a vital target (Scheme 3). Formation of both compounds $5 \& 4$ 
<smiles>O=C1Nc2ccc([N+](=O)[O-])cc2C1=O</smiles><smiles>C[AsH3]O[Mg]</smiles><smiles>O=C(Nc1ccc([N+](=O)[O-])cc1-c1n[nH]c(=S)nc1O)C(F)(F)F</smiles>

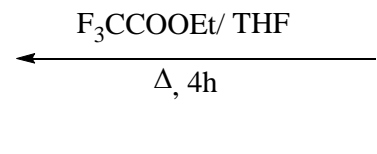<smiles>Nc1ccc([N+](=O)[O-])cc1C(=O)C(=O)O[Na]</smiles><smiles>NNC(N)=S</smiles><smiles>Nc1ccc([N+](=O)[O-])cc1-c1n[nH]c(=S)[nH]c1=O</smiles>

Scheme 1. Synthesis of compounds 1 and 2.<smiles>O=C(Nc1ccc([N+](=O)[O-])cc1C1=NNC(S)N=C1O)C(F)(F)F</smiles>

$\mathrm{NH}_{3} / \mathrm{EtOH}$

$\Delta, 6 \mathrm{~h}$<smiles>Nc1nnc(-c2cc([N+](=O)[O-])ccc2NC(=O)C(F)(F)F)c(O)n1</smiles>

$\Delta, 1 \mathrm{~h}$ $\mathrm{AcOH}$<smiles>[X]c1cccc(C=O)c1</smiles><smiles>[X]c1ccc(C=Nc2nnc(-c3cc([N+](=O)[O-])ccc3NC(=O)C(F)(F)F)c(O)n2)cc1</smiles>

$\mathrm{X}: \mathbf{a}, o-\mathrm{NO}_{2} ; \mathbf{b}, m-\mathrm{NO}_{2} ; \mathbf{c}, \mathrm{NO}_{2} ; \mathbf{d}, p-\mathrm{Br} ; \mathbf{e}, p-\mathrm{Cl} ; \mathbf{f}, p-\mathrm{F}$

Scheme 2. Synthesis of compounds 3 and 4a-f. 
<smiles>[X]c1ccc(C=Nc2nnc(-c3cc([N+](=O)[O-])ccc3NC(F)(F)F)c(O)n2)cc1</smiles><smiles></smiles>

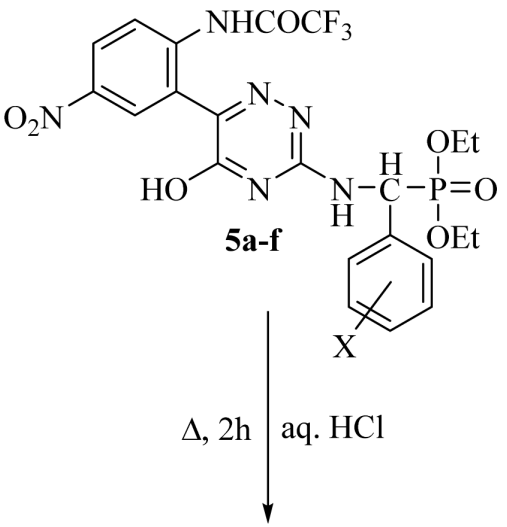<smiles>[X]c1ccc(C(Nc2nnc(-c3cc([N+](=O)[O-])ccc3NC(=O)O)c(O)n2)P(=O)(O)O)cc1</smiles>

$\mathrm{X}: \mathbf{a}, o-\mathrm{NO}_{2} ; \mathbf{b}, m-\mathrm{NO}_{2} ; \mathbf{c}, \mathrm{NO}_{2} ; \mathbf{d}, p-\mathrm{Br} ; \mathbf{e}, p-\mathrm{Cl} ; \mathbf{f}, p-\mathrm{F}$

Scheme 3. Synthesis of compounds 5a-f and 6a-f.

may as they shown in Figure 1.

\section{Result and Discussion}

The former structures of novel fluorinated $\alpha$-amino phosphonic acids have been deduced from correct their elemental analysis and spectral measurements. IR spectra of both the compounds 1-6 recorded the absorption bands $\bar{v}$ at 1530, $1350 \mathrm{~cm}^{-1}$ for asymmetric and symmetric $\mathrm{NO}_{2}$ groups, $3200-3100$ and $1660 \mathrm{~cm}^{-1}$ for $\mathrm{NH}$ and $\mathrm{C}=\mathrm{O}$ of 1,2,4-triazine, also $\bar{v}$ at $1630 \mathrm{~cm}^{-1}$ attribute to $\underline{\mathrm{CONH}}$ group and $1250 \mathrm{~cm}^{-1}$ of C-F functional groups. IR of compound 3 showed $\bar{v}$ at 3300 and $1610 \mathrm{~cm}^{-1}$ stretching and bending of $\mathrm{NH}_{2}$ group, which lacks in all the compounds 4-6. Also, the presence of $\bar{v}$ at $1600-1580 \mathrm{~cm}^{-1}$ for the exocyclic 


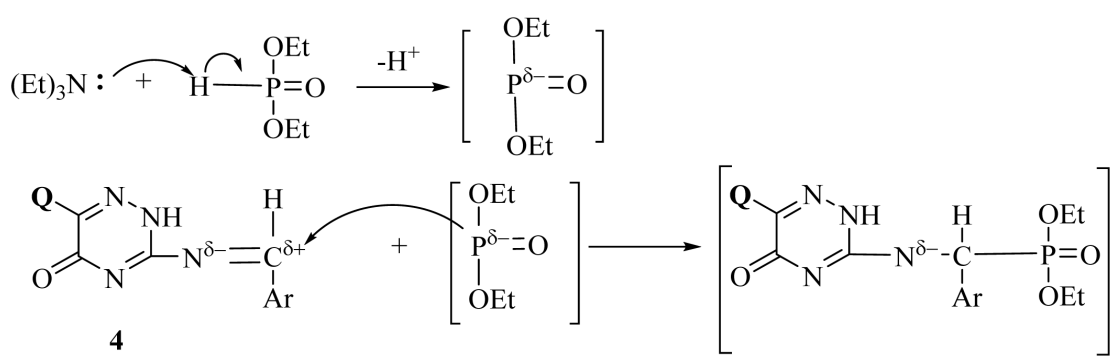
4<smiles>[X]c1cc(C)cc(C)c1NC(=O)Nc1ccc([N+](=O)[O-])cc1C</smiles><smiles></smiles>
5

Figure1. Formation of compound 5 from 4.

$\mathrm{CH}=\mathrm{N}$ group in the compound 4. New functional groups at $\bar{v} 1220-1215(\mathrm{P}=\mathrm{O})$ and 1050 (P-O-R) $\mathrm{cm}^{-1}$ observed in the spectrum of 5 . In addition, showed $\bar{v}$ at $2900-2880$ and $1480-1440 \mathrm{~cm}^{-1}$ for stretching and bending of $\mathrm{CH}_{3} \& \mathrm{CH}_{2}$. On the other hand, IR spectrum of 6 showed $\bar{v}$ at $2730,2680 \mathrm{~cm}^{-1}$ attribute for two hydroxy groups bonded to the phosphorus atom. All the fluorinated 1,2,4-triazinones 2 - 6 showed a stable true hydroxy group at the position-5 of 1,2,4-triazines at $\bar{v} 3500-3450 \mathrm{~cm}^{-1}$. Presence of these hydroxy groups may be due to a large withdrawing from both $\mathrm{NO}_{2}, \mathrm{CF}_{3}$ groups via a type of $\mathrm{H}$-bonding.<smiles>O=C(Nc1ccc([N+](=O)[O-])cc1-c1nnc(S)nc1OP)C(F)(F)F</smiles>

The H-bonding form's of compound 2.

${ }^{1} \mathrm{H}$ NMR spectra of the novel fluorinated $\alpha$-amino phosphonic acids give us a good indication of what those structures. Thus, ${ }^{1} \mathrm{H}$ NMR spectra of compound 1 exhibit $\delta$ at 3.5, 13.0, $11.8 \mathrm{ppm}$ for $\mathrm{NH}_{2}, \mathrm{NH}, \mathrm{NH}$ of 1,2,4-triazinone, in addition to $\delta 8.8,8.2,7.9$ ppm for aromatic protons. ${ }^{1} \mathrm{H}$ NMR spectra of $2 \& 4$ recorded a lack's of $\mathrm{NH}_{2}$ protons while showed $\delta$ at $9.22 \mathrm{ppm}$ for methin proton $(-\mathrm{CH}=\mathrm{N}-)$ in compound 4. Also, ${ }^{1} \mathrm{H}$ NMR spectrum of compound 5 showed a new resonated signal at $\delta 1.2(\mathrm{~J}=6.8 \mathrm{~Hz})$ and large signal $4.0-3.9 \mathrm{ppm}$ for $\mathrm{OCH}_{2} \mathrm{CH}_{3}$ 
protons, with a broad signal at $3.0-2.95,2.7,2.5$ and $1.05,1.03 \mathrm{ppm}$ for $\mathrm{CH}_{2}$ \& $\mathrm{CH}_{3}$ protons. ${ }^{1} \mathrm{H}$ NMR spectrum of compound $6 \mathbf{f}$ showed lacks both $\mathrm{NH}$ protons which is due to a type of $\mathrm{F} . . . \mathrm{H}$ bond while reporting the signal at $4.95 \mathrm{ppm}$ attribute to $\mathrm{OH}$ proton. ${ }^{1} \mathrm{H}$ NMR spectra of all new synthetic compounds 1 - $\mathbf{6}$ recorded the $\delta$ at $11.8 \mathrm{ppm}$ for internal NH of 1,2,4-triazine at position-4, and $8.55 \mathrm{ppm}$ for $\mathrm{NHCO}$ protons. ${ }^{31} \mathrm{P}$ NMR (DMSO) of the $6 \mathrm{f}$ exhibit resonated signals at $\delta 14.0(\mathrm{O}=\mathrm{P}-\mathrm{OH})$ and $20.5(\mathrm{P}-\mathrm{CH}) \mathrm{ppm}$. Also, ${ }^{1} \mathrm{H}$ NMR spectra of 6 recorded the P-CHAr proton at tow doublets at $4.55\left(\mathrm{~J}_{\mathrm{PCH}}=21 \mathrm{~Hz}\right)$ and $4.64\left(\mathrm{~J}_{\mathrm{PCH}}=\right.$ $18 \mathrm{~Hz}) \mathrm{ppm}$ while that showed the $\mathrm{P}-\mathrm{OH}$ protons at $3.00 \mathrm{ppm}$ which supported the existence of that structures. ${ }^{13} \mathrm{C}$ NMR spectrum of compound 5 supported their structure due to the presence of the characteristic carbon atoms at $\delta 16$, 60.1, 45.2 and $177.5 \mathrm{ppm}$ attributed to $\mathrm{CH}_{3}, \mathrm{CH}_{2}, \mathrm{CH}-\mathrm{P}$ and $\mathrm{C}=\mathrm{O}$ of 1,2,4-triazines. In addition, signals at $\delta 155$ and $130-127 \mathrm{ppm}$ for $\mathrm{CONH}$ and aromatic carbons. The aliphatic carbons $\mathrm{CH}_{2}, \mathrm{CH}_{3}$ of compound 5 disappeared in that of compound 6. Finally, mass spectrometry study of novel fluorinated $\alpha$-amino phosphonic acids, for example, 6 band $6 \mathrm{f}$ recorded a molecular ion fragments at low intensity, with base peaks at m/e 231 for (6b) and m/e 95 for (6f) attribute to $\alpha$-amino phosphonic radicals (Figure $2 \&$ Figure 3 ).

The higher stability of their base peak may be due to the tautomeric forms present and the free delocalization from $\mathrm{HN}$ to $\mathrm{P}=\mathrm{O}$ centers (Figure 4).

\section{Experimental}

The melting point recorded on Stuart scientific SMP3 (Bibby, UK) melting point

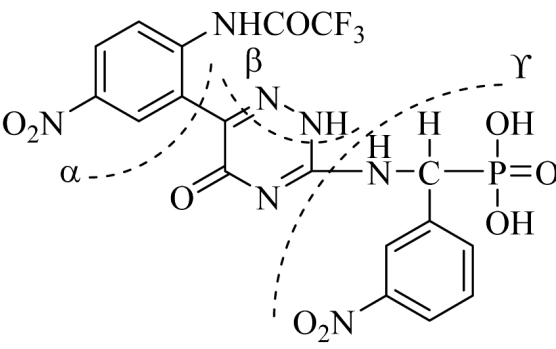

6b, M/e (550, $\left.\mathrm{M}+\mathrm{H}_{2} \mathrm{O}, 1.0 \%\right)$

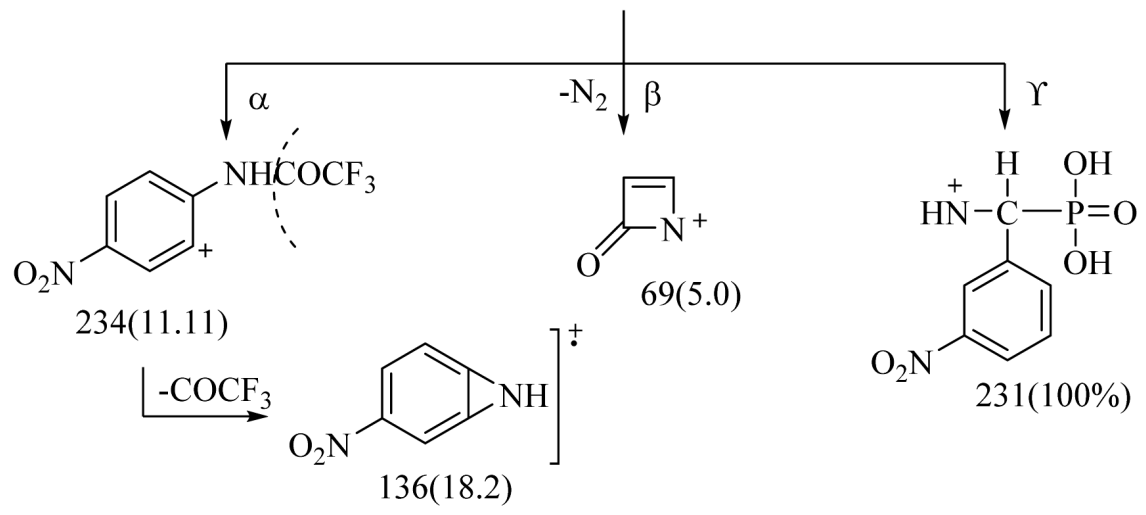

Figure 2. Mass fragmentation pattern of compound $6 \mathrm{~b}$. 


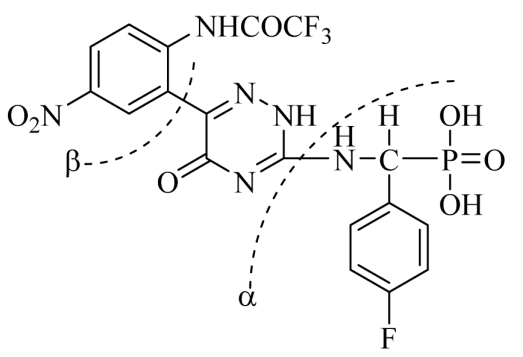

6f, $\mathrm{M} / \mathrm{e}(534, \mathrm{M}+2,5.18 \%)$

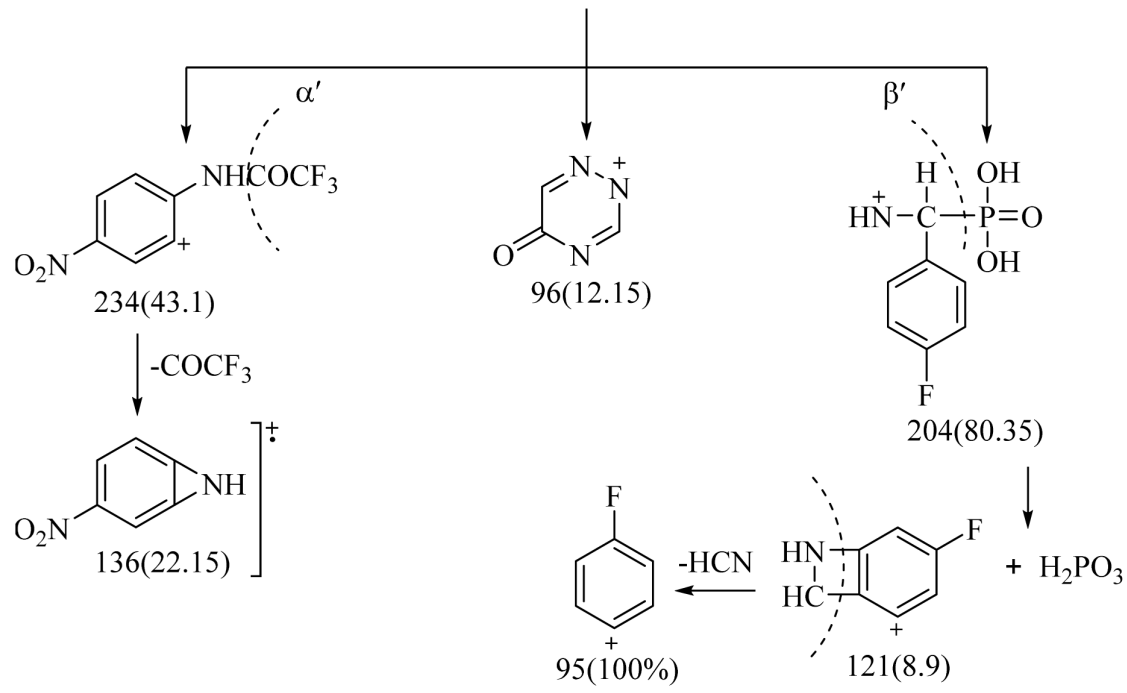

Figure 3. Mass fragmentation pattern of compound $6 f$.

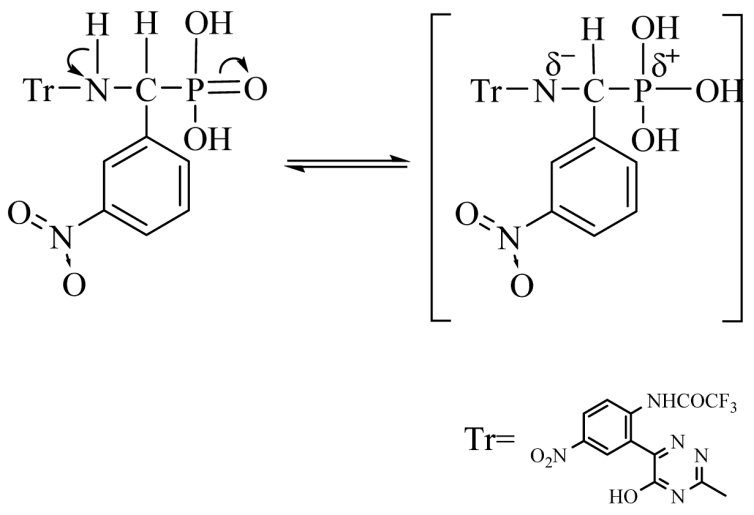

Figure 4. The stability of $\alpha$-amino phosphonic acids 6 b.

apparatus and reported as uncorrected. A Perkin Elmer (Lambda EZ-2101) double beam spectrophotometer $(190-1100 \mathrm{~nm})$ used for recording the electronic spectra. A Perkin Elmer model RXI-FT-IR $55,529 \mathrm{~cm}^{-1}$ used for recording the IR spectra. A Brucker advance DPX $400 \mathrm{MHz}$ using TMS as an internal standard for recording the ${ }^{1} \mathrm{H} /{ }^{13} \mathrm{C}$ NMR spectra in deuterated DMSO ( $\delta$ in ppm). AGC-MS-QP 1000 Ex model used for recording the mass spectra. Hexafluorobenzene used as an external standard for ${ }^{19} \mathrm{FNMR}$ at $84.25 \mathrm{MHz}$ and ${ }^{31} \mathrm{P}$ (in 
$\mathrm{CDCl}_{3}, 101.25 \mathrm{MHZ}$ ). Elemental analysis performed on Micro Analytical Center of National Reaches Center-Dokki, Cairo, Egypt.

6-(2'-Amino-5'-nitrophenyl)-3-thioxo-1,2,4-triazin-5 (2H,4H)one (1) [26]

A mixture of 5 -nitroisatin $(0.1 \mathrm{~mol}$, in $100 \mathrm{ml}$ of $5 \%$ aq. $\mathrm{NaOH})$ and thiosemicarbazide $(0.1 \mathrm{~mol}$, in $10 \mathrm{ml}$ hot water) refluxed for $2 \mathrm{~h}$, cooled then poured onto ice-AcOH. The solid produced filtered off, and crystallization from $\mathrm{MeOH}$ to give compound 1 as reddish brown solid, yield $85 \%$, m.p. $290^{\circ} \mathrm{C}-291^{\circ} \mathrm{C}$. IR spectrum $\bar{v}\left(\mathrm{~cm}^{-1}\right)$ : 3255(NH), 3138(NH), 3082(NH), 3020(aromatic $\left.\mathrm{CH}\right), 1608$ (binding $\mathrm{NH}_{2}$ ), 1595(C=N), 1509, 1310(asym. \& sym. $\mathrm{NO}_{2}$ ), 1173(C=S), 837, 817, 750 (aromatic CH). Calculated $\mathrm{C}_{9} \mathrm{H}_{7} \mathrm{~N}_{5} \mathrm{O}_{3} \mathrm{~S}\left(\mathrm{M}^{+}\right.$265): C, 40.75; H, 2.66; N, 26.40; S, 12.09\%. Found: C, 40.34; H, 2.60; N, 26.31; S, 11.99\%.

2,2,2-Trifluoro-N-[2-(5-hydroxy-3-thioxo-2,3-dihydro-1,2,4-triazin-6-yl)4-nitrophenyl]acetamide (2)

Equimolar amounts of compound 1 and ethyl 2,2,2-trifluoroacetate in THF $(100 \mathrm{ml})$ refluxed for $4 \mathrm{~h}$, cooled. The solid obtained filtered off and crystallized from $\mathrm{EtOH}$ to give compound 2 as deep green solid, yield 76\%, m.p: $270^{\circ} \mathrm{C}-272^{\circ} \mathrm{C}$. IR spectrum $\bar{v}\left(\mathrm{~cm}^{-1}\right): 3448(\mathrm{OH}), 3324, \quad 3191(\mathrm{NH}), \quad 3080($ aromatic $\mathrm{CH})$, 1703 $\left(\mathrm{COCF}_{3}\right), 1615(\mathrm{C}=\mathrm{N}), 1519,1321$ (asym. \& sym. $\left.\mathrm{NO}_{2}\right), 1242(\mathrm{C}-\mathrm{F}), 850,810$, 780 (aromatic CH). ${ }^{1} \mathrm{H}$ NMR(400 MHz, DMSO-d $\left.\mathrm{d}_{6}\right) \delta(\mathrm{ppm}): 14.64(\mathrm{~s}, 1 \mathrm{H}, \mathrm{OH})$, 13.72(s, $1 \mathrm{H}, \mathrm{NH}), 12.45(\mathrm{~s}, 1 \mathrm{H}, \mathrm{NH}), 9.1,8.9,8.5,8.3(\mathrm{~m}, 4 \mathrm{H}$, aromatic protons), 3.56(s, OH) $.{ }^{13} \mathrm{C} \operatorname{NMR}\left(100 \mathrm{MHz}, \mathrm{DMSO}_{6}\right) \quad \delta(\mathrm{ppm}): 173.32(\mathrm{C}=\mathrm{S}), 153.61$, 153.75(2C=O), 145.7, 143.10(C-F), 135.07(NCN), 128.0-121(aromatic carbons), 118.47, 114.54, 113.52 $\left(\mathrm{C}_{5}, \mathrm{C}_{6}\right.$ of 1,2,4-triazine). Calculated $\mathrm{C}_{11} \mathrm{H}_{6} \mathrm{~F}_{3} \mathrm{~N}_{5} \mathrm{O}_{4} \mathrm{~S}\left(\mathrm{M}^{+}\right.$ 361): C, 36.57; H, 1.67; F, 15.78; N, 19.39; S, 8.87\%. Found: C, 36.40; H, 1.61; F, $15.49 ; \mathrm{N}, 19.15 ; \mathrm{S}, 8.75 \%$.

3-Amino-6-[2'-(trifluoroacetamido-5 "-nitrophenyl)]-1,2,4-triazin-5(4H) one (3)

A mixture of $2(0.1 \mathrm{~mol})$ and a liquid $\mathrm{NH}_{3}(20 \mathrm{ml}, 39 \%)$, with ethanol $(100 \mathrm{ml})$, refluxed $6 \mathrm{~h}$, cooled then poured onto ice-drops $\mathrm{AcOH}$. The resulting solid, filtered off and crystallized from EtOH to give light green solid, yield 86\%, m.p: $305^{\circ} \mathrm{C}-307^{\circ} \mathrm{C}$. IR spectrum $\bar{v}\left(\mathrm{~cm}^{-1}\right): 3448(\mathrm{OH}), 3323(\mathrm{NH}), 3090(\mathrm{NH}), 1702$ $(\mathrm{C}=\mathrm{O}), 1615$ (deformation $\left.\mathrm{NH}_{2}\right), 1557(\mathrm{C}=\mathrm{N}), 1531,1312$ (asym. \& sym. $\mathrm{NO}_{2}$ ), 1241(C-F), 988, 830, 749(aromatic CH), 607(C-F). ${ }^{1} \mathrm{H}$ NMR(400 MHz, DMSO-d 6 ) $\delta(\mathrm{ppm}): 13.59,12.41$ (each s, $2 \mathrm{NH}), 9.1(1 \mathrm{H}, \mathrm{OH}$-triazine), $8.95-8.60,8.53-8.35$ (each d, d, $2 \mathrm{H}$, aromatic adjacent of $\left.\mathrm{NO}_{2}\right), 8.27-8.01,7.99-6.76(\mathrm{~d}, \mathrm{~d} 2 \mathrm{H}$, aromatic), $3.44\left(\mathrm{~s}, 2 \mathrm{H}, \mathrm{NH}_{2}\right)$, protons. ${ }^{13} \mathrm{C} \mathrm{NMR}\left(100 \mathrm{MHz}, \mathrm{DMSO}-\mathrm{d}_{6}\right) \delta(\mathrm{ppm})$ : 173.3(C=O), 153, 152(C-O), 145(C-F), 153(NCN), 128 - 121(aromatic carbons), 114, 113(triazine). Calculated $\mathrm{C}_{11} \mathrm{H}_{7} \mathrm{~F}_{3} \mathrm{~N}_{6} \mathrm{O}_{4}\left(\mathrm{M}^{+} 344\right)$ : C, 38.38; H, 2.05; F, 16.56; N, 24.42\%. Found: C, 38.18; H, 1.99; F, 16.36; N, 24.21\%.

\section{Schiff bases $4 \mathrm{a}-\mathrm{f}$}

A mixture of $3(0.01 \mathrm{~mol})$ and $(o-, m-, p$-nitrobenzaldehydes, $p$-bromo, $p$-chloro, and $p$-fluoro benzaldehydes) $(0.01 \mathrm{ml})$ refluxed in $\mathrm{AcOH}(50 \mathrm{ml})$ for $1 \mathrm{~h}$, cooled then poured onto ice. The yielded solids filtered off and crystallized from a suit- 
able solvent (EtOH, $\mathrm{MeOH} \&$ Isopropyl alcohol) to give $4 \mathrm{a}-\mathrm{f}$.

4a: Brown green solid, yield $83 \%$, m.p: $270^{\circ} \mathrm{C}-272^{\circ} \mathrm{C}$. IR spectrum $\bar{v}\left(\mathrm{~cm}^{-1}\right)$ : 3447( (OH), 3323 $(\mathrm{NH}), 3080$ (aromatic $\mathrm{CH}), 1703(\mathrm{C}=\mathrm{O}), 1615(\mathrm{C}=\mathrm{C}), 1557(\mathrm{C}=\mathrm{N})$, 1479(exo $\mathrm{CH}=\mathrm{N}), 1519,1322$ (asym., sym. $\mathrm{NO}_{2}$ ), 1270(C-F), 927, 831, 749(aromatic $\mathrm{CH}$ ), 607(C-F). Calculated $\mathrm{C}_{18} \mathrm{H}_{10} \mathrm{~F}_{3} \mathrm{~N}_{7} \mathrm{O}_{6}\left(\mathrm{M}^{+}\right.$477): $\mathrm{C}, 45.29 ; \mathrm{H}$, 2.11; F, 11.94; N, 20.54\%. Found: C, 44.78; H, 2.08; F, 11.79; N, 20.27\%.

4b: Deep brown solid, yield 76\%, m.p: $268^{\circ} \mathrm{C}-270^{\circ} \mathrm{C}$. IR spectrum $\bar{v}\left(\mathrm{~cm}^{-1}\right)$ : 3446(OH), 3322.55(NH), 3083(aromatic $\mathrm{CH}), 1702.56(\mathrm{C}=\mathrm{O}), 1615.21(\mathrm{C}=\mathrm{N})$, 1531, 1307 (asym., sym. $\mathrm{NO}_{2}$ ), 1478( $\left.\mathrm{CH}=\mathrm{N}\right), 1453,1427$ (deformation $\left.\mathrm{CH}=\mathrm{N}\right)$, 1270(C-F), 1239(C-F), 987, 928, 830, 749(aromatic CH), 607(C-F). ${ }^{1} \mathrm{H}$ NMR(400 MHz, DMSO-d $\left.\mathrm{d}_{6}\right) \delta(\mathrm{ppm}): 10.13(\mathrm{~s}, 1 \mathrm{H}, \mathrm{NH}), 12.42-12.23(\mathrm{NHCO}), 10.13(\mathrm{~s}, 1 \mathrm{H}$, $\mathrm{CH}=\mathrm{N}$ ), $9.2-8.5,8.4-6.75$ (each $\mathrm{d}, \mathrm{d}, 8 \mathrm{H}$, aromatic protons), 3.48(s, $1 \mathrm{H}, \mathrm{OH})$. ${ }^{13} \mathrm{C}$ NMR(100 MHz, DMSO-d $\left.{ }_{6}\right) \delta(\mathrm{ppm}): 173(\mathrm{C}=\mathrm{O}), 153(\mathrm{CONH}), 145(\mathrm{C}-\mathrm{F})$, $137(\mathrm{CH}=\mathrm{N}), 130-123$ (aromatic carbons), 114, 113(carbons of 1,2,4-triazine). Calculated $\mathrm{C}_{18} \mathrm{H}_{10} \mathrm{~F}_{3} \mathrm{~N}_{7} \mathrm{O}_{6}\left(\mathrm{M}^{+} 477\right): \mathrm{C}, 45.29 ; \mathrm{H}, 2.11 ; \mathrm{F}, 11.94 ; \mathrm{N}, 20.54 \%$. Found: C, 44.89; H, 2.00; F, 11.81; N, 20.39\%.

4c: Brown green solid, yield $88 \%$, m.p: $274^{\circ} \mathrm{C}-276^{\circ} \mathrm{C}$ IR spectrum $\bar{v}\left(\mathrm{~cm}^{-1}\right)$ : 3447(OH), 3324(NH), 3084(aromatic $\mathrm{CH}), 1703(\mathrm{C}=\mathrm{O}), 1615(\mathrm{C}=\mathrm{C}), 1557(\mathrm{C}=\mathrm{N})$, 1479(exo $\mathrm{CH}=\mathrm{N}$ ), 1531, 1322(asym., sym. $\mathrm{NO}_{2}$ ), 1270(C-F), 900, 880, 850 (aromatic $\mathrm{CH}), 610(\mathrm{C}-\mathrm{F})$. Calculated $\mathrm{C}_{18} \mathrm{H}_{10} \mathrm{~F}_{3} \mathrm{~N}_{7} \mathrm{O}_{6}\left(\mathrm{M}^{+}\right.$477): $\mathrm{C}$, 45.29; $\mathrm{H}, 2.11 ; \mathrm{F}$, 11.94; N, 20.54\%. Found: C, 44.71; H, 2.01; F, 11.64; N, 20.12\%.

4d: Brown solid, yield 79\%, m.p: $260^{\circ} \mathrm{C}-262^{\circ} \mathrm{C}$. IR spectrum $\bar{v}\left(\mathrm{~cm}^{-1}\right): 3448$ $(\mathrm{OH}), \quad 3324(\mathrm{NH}), \quad 3085(\mathrm{NH}), \quad 1704(\mathrm{C}=\mathrm{O}), \quad 1616(\mathrm{C}=\mathrm{C}), 1558(\mathrm{C}=\mathrm{N}), \quad 1480($ exo $\mathrm{CH}=\mathrm{N}$ ), 1515, 1324(asym. \& sym. $\mathrm{NO}_{2}$ ), 1272(C-F), 988, 929, 749(aromatic $\mathrm{CH}$ ), 690(C-Br), 640(C-F). Calculated $\mathrm{C}_{18} \mathrm{H}_{10} \mathrm{BrF}_{3} \mathrm{~N}_{6} \mathrm{O}_{4}\left(\mathrm{M}^{+}\right.$509): C, 42.29; H, 1.97; Br, 15.63; F, 11.15; N, 16.44\%. Found: C, 41.97; H, 1.71; Br, 15.54; F, 10.99; N, $16.24 \%$.

4e: Brownish yellow solid, yield $77 \%$, m.p: $267^{\circ} \mathrm{C}-269^{\circ} \mathrm{C}$. IR spectrum $\bar{v}\left(\mathrm{~cm}^{-1}\right)$ : 3447( $\left.\mathrm{OH}\right), 3320(\mathrm{NH}), 3089(\mathrm{NH}), 1701(\mathrm{C}=\mathrm{O}), 1614(\mathrm{C}=\mathrm{C}), 1553(\mathrm{C}=\mathrm{N})$, 1477(exo $\mathrm{CH}=\mathrm{N}), 1516,1322$ (asym. \& sym. $\mathrm{NO}_{2}$ ), 1269.9(C-F), 986, 929, 749.7(aromatic CH), 688(C-Cl), 646(C-F). Calculated $\mathrm{C}_{18} \mathrm{H}_{10} \mathrm{ClF}_{3} \mathrm{~N}_{6} \mathrm{O}_{4}\left(\mathrm{M}^{+} 466\right)$ : C, 46.32; H, 2.16; Cl, 7.59; F, 12.21; N, 18.01\%. Found: C, 45.89; H, 2.13; Cl, 7.28; F, 11.96; N, 17.71\%.

4f: Reddish brown solid, yield 84\%, m.p: $271^{\circ} \mathrm{C}-273^{\circ} \mathrm{C}$. IR spectrum $\bar{v}\left(\mathrm{~cm}^{-1}\right)$ : 3446(OH), 3321(NH), 3081 $(\mathrm{NH}), 1702(\mathrm{C}=\mathrm{O}), 1557(\mathrm{C}=\mathrm{N}), 1517,1320$ (asym. \& sym. $\mathrm{NO}_{2}$ ), 1239(C-F), 928, 931, 749(aromatic CH), 647(C-F). ${ }^{1} \mathrm{H}$ NMR(400 $\left.\mathrm{MHz}, \mathrm{DMSO}-\mathrm{d}_{6}\right) \delta(\mathrm{ppm}): 13.44(\mathrm{~s}, 1 \mathrm{H}, \mathrm{NH}), 9.52(\mathrm{~s}, 1 \mathrm{H}, \mathrm{CH}=\mathrm{N}), 8.35-7.99 \&$ 7.01 - 6.75(each $\mathrm{d}, \mathrm{d} 7 \mathrm{H}$, aromatic protons), $3.41\left(\mathrm{~s}, 1 \mathrm{H}, \mathrm{OH}\right.$ of $\mathrm{C}_{5}-1,2,4$-triazine). ${ }^{13} \mathrm{C}$ NMR(100 MHz, DMSO-d $\left.{ }_{6}\right) \delta(\mathrm{ppm}): 173(\mathrm{C}=\mathrm{O}), 153,152(\mathrm{C}-\mathrm{OR}), 145.7(\mathrm{C}-\mathrm{F})$, 135 (NCN of 1,2,4-triazine), 128-126(aromatic carbons), 114, 113( $\mathrm{C}_{5}, \mathrm{C}_{6}$ of 1,2,4-triazine). Calculated $\mathrm{C}_{18} \mathrm{H}_{10} \mathrm{~F}_{4} \mathrm{~N}_{6} \mathrm{O}_{4}\left(\mathrm{M}^{+} 450\right): \mathrm{C}, 48.01 ; \mathrm{H}, 2.24 ; \mathrm{F}, 16.88 ; \mathrm{N}$, $18.66 \%$. Found: C, 47.83 ; H, 2.19; F, 16.66; N, 18.46\%.

Diethyl [6-(2'-trifluoroacetamido-5'-nitrophenyl)-5-hydroxy-1,2,4-triazin3-yl]-amino-(aryl) methyl phosphonates (5a-f) 
A mixture of $4 \mathrm{a}-\mathbf{e}$ and/ or $4 \mathrm{f}(0.01 \mathrm{~mol})$ and diethyl phosphonate $(0.01 \mathrm{~mol})$ in few drops of TEA, fused at $80^{\circ} \mathrm{C}-100^{\circ} \mathrm{C}$ for $6-8 \mathrm{~h}$, cooled the treated with dioxan. The solid obtained crystallized from a suitable solvent to give $5 \mathrm{a}-\mathrm{f}$.

5a: Deep brown solid, yield 82\%, m.p: $263^{\circ} \mathrm{C}-265^{\circ} \mathrm{C}$. IR spectrum $\bar{v}\left(\mathrm{~cm}^{-1}\right)$ : 3447( (OH), 3321 (NH), 3085(aromatic $\mathrm{CH}), 2970($ aliphatic $\mathrm{CH}), 1701(\mathrm{C}=\mathrm{O})$, 1615(C=N), 1532, 1309 (asym., sym. $\mathrm{NO}_{2}$ ), 1481, 1428(deformation $\mathrm{CH}_{2}, \mathrm{CH}_{3}$ ), 1237(C-F), 1159(P=O), $1100(\mathrm{O}-\mathrm{P}-\mathrm{O}-\mathrm{R}), 840$, 805(aromatic CH), 610(C-F). Calculated $\mathrm{C}_{22} \mathrm{H}_{21} \mathrm{~F}_{3} \mathrm{~N}_{7} \mathrm{O}_{9} \mathrm{P}\left(\mathrm{M}^{+}\right.$615): $\mathrm{C}, 42.94 ; \mathrm{H}, 3.44 ; \mathrm{F}, 9.26 ; \mathrm{N}, 15.93 ; \mathrm{P}, 5.03 \%$. Found: C, 42.59; H, 3.38; F, 9.15; N, 15.70; P, 4.93\%.

5b: Deep brown solid, yield 78\%, m.p: $260^{\circ} \mathrm{C}-262^{\circ} \mathrm{C}$. IR spectrum $\bar{v}\left(\mathrm{~cm}^{-1}\right)$ : 3433(OH), 3316(NH), 3083(aromatic $\mathrm{CH}), 2969$ (aliphatic $\mathrm{CH}), 1698(\mathrm{C}=\mathrm{O})$, 1616 $\left(\mathrm{C}=\mathrm{N}\right.$ ), 1532, 1311 (asym., sym. $\mathrm{NO}_{2}$ ), 1481, 1428(deformation $\mathrm{CH}_{2}, \mathrm{CH}_{3}$ ), 1245(C-F), 1159(P=O), 1098(O-P-O-R), 860, 810(aromatic CH), 600(C-F). ${ }^{1} \mathrm{H}$ NMR(400 MHz, DMSO-d $\left.\mathrm{d}_{6}\right) \delta(\mathrm{ppm}): 13.59(\mathrm{NH}), 8.37(\mathrm{CH}-\mathrm{NH}), 8.36-8.0$, 7.99 - 6.76(each d, d, aromatic protons), 3.82(s, $1 \mathrm{H}, \mathrm{OH}), 3.76-3.44(\mathrm{~b}, \mathrm{NH})$, 2.89, $2.53 \& 1.05,1.03$ (each s, $\left.2 \mathrm{CH}_{2} \& \mathrm{CH}_{3}\right) .{ }^{13} \mathrm{C} \mathrm{NMR}\left(100 \mathrm{MHz}, \mathrm{DMSO}-\mathrm{d}_{6}\right.$ ) $\delta(\mathrm{ppm}): 173.3(\mathrm{C}=\mathrm{O}), 153,152(\mathrm{C}-\mathrm{OR}), 145.80(\mathrm{C}-\mathrm{F}), 135(\mathrm{NCN}), 128$ - 126(aromatic carbons), 114, 113.59( $\mathrm{C}_{5}, \mathrm{C}_{6}$ of 1,2,4-triazine), 38.30(carbons of $\left.\mathrm{CH}_{2}, \mathrm{CH}_{3}\right)$. Calculated $\mathrm{C}_{22} \mathrm{H}_{21} \mathrm{~F}_{3} \mathrm{~N}_{7} \mathrm{O}_{9} \mathrm{P}\left(\mathrm{M}^{+}\right.$615): $\mathrm{C}, 42.94 ; \mathrm{H}, 3.44 ; \mathrm{F}, 9.26 ; \mathrm{N}, 15.93 ; \mathrm{P}, 5.03 \%$. Found: C, 42.66; H, 3.25; F, 9.11; N, 15.78; P, 4.85\%.

5c: Brown sold, yield $87 \%$, m.p: $270^{\circ} \mathrm{C}-272^{\circ} \mathrm{C}$. IR spectrum $\bar{v}\left(\mathrm{~cm}^{-1}\right)$ : $3446(\mathrm{OH}), 3320(\mathrm{NH}), 3082($ aromatic $\mathrm{CH}), 2970$ (aliphatic $\mathrm{CH}), 1700(\mathrm{C}=\mathrm{O})$, 1614(C=N), 1528, 1310(asym., sym. $\mathrm{NO}_{2}$ ), 1477, 1427 (deformation $\mathrm{CH}_{2}, \mathrm{CH}_{3}$ ), 1238(C-F), 1159(P=O), 1104(O-P-O-R), 850, 810(aromatic CH), 608(C-F). Calculated $\mathrm{C}_{22} \mathrm{H}_{21} \mathrm{~F}_{3} \mathrm{~N}_{7} \mathrm{O}_{9} \mathrm{P}\left(\mathrm{M}^{+}\right.$615): $\mathrm{C}, 42.94 ; \mathrm{H}, 3.44 ; \mathrm{F}, 9.26 ; \mathrm{N}, 15.93 ; \mathrm{P}, 5.03 \%$. Found: C, 42.69; H, 3.44; F, 9.26; N, 15.93; P, 5.03\%.

5d: Deep brown solid, yield 92\%, m.p: $302^{\circ} \mathrm{C}-305^{\circ} \mathrm{C}$. IR spectrum $\bar{v}\left(\mathrm{~cm}^{-1}\right)$ : 3434 $(\mathrm{OH}), 3317(\mathrm{NH}), 3081($ aromatic $\mathrm{CH}), 2970$ (aliphatic $\mathrm{CH}), 1700(\mathrm{C}=\mathrm{O})$, 1553(C=N), 1532, 1311(asym. \& sym. $\mathrm{NO}_{2}$ ), 1479, 1427(deformation aliphatic), 1242(C-F), 1223(P=O), $1100(\mathrm{P}-\mathrm{O}-\mathrm{Et})$ 987, 780, 749(aromatic $\mathrm{CH}$ ), 700(C-Br), 605(C-F). Calculated, $\mathrm{C}_{22} \mathrm{H}_{21} \mathrm{BrF}_{3} \mathrm{~N}_{6} \mathrm{O}_{7} \mathrm{P}\left(\mathrm{M}^{+}\right.$649): $\mathrm{C}, 40.70 ; \mathrm{H}, 3.26 ; \mathrm{Br}, 12.31 ; \mathrm{F}$, 8.78; N, 12.94; P, 4.77\%. Found: C, 40.39; H, 3.11; Br, 12.16; F, 8.61; N, 12.80; P, $4.66 \%$.

5e: Black brown solid, yield $86 \%$, m.p: $296^{\circ} \mathrm{C}-298^{\circ} \mathrm{C}$. IR spectrum $\bar{v}\left(\mathrm{~cm}^{-1}\right)$ : $3434(\mathrm{OH}), 3317(\mathrm{NH}), 3084($ aromatic $\mathrm{CH}), 2970($ aliphatic $\mathrm{CH}), 1700(\mathrm{C}=\mathrm{O})$, 1553(C=N), 1532, 1311(asym. \& sym. $\mathrm{NO}_{2}$ ), 1480, 1427(deformation aliphatic), $1242(\mathrm{C}-\mathrm{F}), 1210(\mathrm{P}=\mathrm{O}), 1099(\mathrm{P}-\mathrm{O}-\mathrm{Et})$ 987, 780, 749(aromatic CH), 730(C-Cl), 600(C-F). Calculated $\mathrm{C}_{22} \mathrm{H}_{21} \mathrm{ClF}_{3} \mathrm{~N}_{6} \mathrm{O}_{7} \mathrm{P}\left(\mathrm{M}^{+}\right.$604): $\mathrm{C}$, 43.69; H, 3.50; Cl, 5.86; F, 9.42; N, 13.89; P, 5.12\%. Found: C, 43.48; H, 3.43; Cl, 5.57; F, 9.22; N, 13.69; P, $4.98 \%$.

5f: Light brown solid, yield $78 \%$, m.p: $285^{\circ} \mathrm{C}-287^{\circ} \mathrm{C}$. IR spectrum $\bar{v}\left(\mathrm{~cm}^{-1}\right)$ : 3447(OH), 3284(NH), 3198(NH), 3084(aromatic CH), 2970(aliphatic $\mathrm{CH}$ ), 1695(C=O), 1556(C=N), 1516, 1304(asym. \& sym. $\mathrm{NO}_{2}$ ), 1478, 1427(deformation 
aliphatic), 1240(C-F), 1222(P=O), 1070(P-O-Et) 983, 787, 749(aromatic $\mathrm{CH}$ ), 608(C-F). ${ }^{1} \mathrm{H} \mathrm{NMR}\left(400 \mathrm{MHz}, \mathrm{DMSO}-\mathrm{d}_{6}\right) \delta(\mathrm{ppm}): 12.25(\mathrm{~s}, 1 \mathrm{H}, \mathrm{NH}), 10.76(\mathrm{~s}, 1 \mathrm{H}$, $\mathrm{OH}), 9.9(\mathrm{~s}, 1 \mathrm{H}, \mathrm{CH}-\mathrm{N}), 9.05-8.01 \& 7.99-6.54$ (each d, d 7H, aromatic protons), 3.44(s, $1 \mathrm{H}, \mathrm{OH}), 2.52,1.24\left(\right.$ each m, $\left.10 \mathrm{H}, \mathrm{O}-\mathrm{CH}_{2} \mathrm{CH}_{3}\right) \cdot{ }^{13} \mathrm{C} \mathrm{NMR}(100 \mathrm{MHz}$, DMSO- $\left._{6}\right) \delta(\mathrm{ppm}): 173(\mathrm{C}=\mathrm{O}), 152(\mathrm{C}-\mathrm{OH}), 150(\mathrm{C}-\mathrm{NH}), 147(\mathrm{C}-\mathrm{F}), 145\left(\mathrm{C}_{6}\right.$ of 1,2,4-triazine), $142\left(\mathrm{C}-\mathrm{NO}_{2}\right), 137\left(\mathrm{C}_{3}\right.$ of 1,2,4-triazine) 130 - 126(aromatic carbons), 116( $\mathrm{Ar}-\underline{\mathrm{CH}}-\mathrm{P}), 113\left(\mathrm{CH}_{2}-\mathrm{O}\right), 40\left(\mathrm{CH}_{3}-\mathrm{CH}_{2}\right)$. Calculated $\mathrm{C}_{22} \mathrm{H}_{21} \mathrm{~F}_{4} \mathrm{~N}_{6} \mathrm{O}_{7} \mathrm{P}\left(\mathrm{M}^{+}\right.$ 588): C, 44.91; H, 3.60; F, 12.92; N, 14.28 P, 5.26\%. Found: C, 44.66; H, 3.58; F, $12.71 ; \mathrm{N}, 14.11 \mathrm{P}, 5.01 \%$.

[((6-(5'-nitro-2'-(2",2",2"-trifluoroacetamido)phenyl)-5-oxo-2,5-dihydro -1,2,4-triazin-3-yl)amino)(phenyl)methyl]phosphonic acids(6a-f)

A mixture of $5(0.01 \mathrm{~mol})$ and dil. $\mathrm{HCl}(10 \mathrm{ml}, 5 \%)$ refluxed for $2 \mathrm{~h}$, cooled, then neutralized with diluted $\mathrm{NaHCO}_{3}$. The solid poured, filtered off and crystallized from suitable solvents to give (6a-f).

6a: Brown solid, yield $85 \%$, m.p: $282^{\circ} \mathrm{C}-284^{\circ} \mathrm{C}$. IR spectrum $\bar{v}\left(\mathrm{~cm}^{-1}\right)$ : 3446(OH), 3321 $(\mathrm{NH}), 3080($ aromatic $\mathrm{CH}), 2970$ (aliphatic $\mathrm{CH}), 1701(\mathrm{C}=\mathrm{O})$, 1614(C=N), 1557, 1305(asym., sym. $\mathrm{NO}_{2}$ ), $1269(\mathrm{C}-\mathrm{F}), 1160(\mathrm{P}=\mathrm{O}), 1090(\mathrm{O}-\mathrm{P}-\mathrm{O})$, 980, 840(aromatic CH), 610(C-F). Calculated $\mathrm{C}_{18} \mathrm{H}_{13} \mathrm{~F}_{3} \mathrm{~N}_{7} \mathrm{O}_{9} \mathrm{P}\left(\mathrm{M}^{+}\right.$559): C, 38.65; H, 2.34; F, 10.19; N, 17.53; P, 5.54\%. Found: C, 38.41; H, 2.08; F, 9.99; N, 17.30; $\mathrm{P}, 5.28 \%$.

6b: Brown solid, yield $94 \%$, m.p: $289^{\circ} \mathrm{C}-291^{\circ} \mathrm{C}$. IR spectrum $\bar{v}\left(\mathrm{~cm}^{-1}\right)$ : 3447( $(\mathrm{OH}), 3323(\mathrm{NH}), 1702(\mathrm{C}=\mathrm{O}), 1615(\mathrm{C}=\mathrm{N}), 1531,1308$ (asym., sym. $\left.\mathrm{NO}_{2}\right)$, 1270(C-F), $1159(\mathrm{P}=\mathrm{O}), 1090(\mathrm{O}-\mathrm{P}-\mathrm{O}), 987,850$ (aromatic $\mathrm{CH}), 606(\mathrm{C}-\mathrm{F}) .{ }^{1} \mathrm{H}$ NMR(400 MHz, DMSO-d $\left.\mathrm{d}_{6}\right) \delta(\mathrm{ppm}): 13.5,13.19,12.24$ (each s, 3NH), 9.0(s, $1 \mathrm{H}$, CH-P), $8.61-8.14,7.98-6.75$ (each $\mathrm{d}, \mathrm{d}, 7 \mathrm{H}$, aromatic protons), 5.95(s, $1 \mathrm{H}$, P-OH). ${ }^{13} \mathrm{C}$ NMR(100 MHz, DMSO-d $\left.\mathrm{d}_{6}\right) \delta(\mathrm{ppm}): 173.3(\mathrm{C}=\mathrm{O}), 153,152(\mathrm{C}-\mathrm{O})$, 145.80(C-F), 135.1(NCN), 128.02 - 126.68(aromatic carbons), 114.48, 113.48 (carbons of 1,2,4-triazine). Calculated $\mathrm{C}_{18} \mathrm{H}_{13} \mathrm{~F}_{3} \mathrm{~N}_{7} \mathrm{O}_{9} \mathrm{P}\left(\mathrm{M}^{+}\right.$559): $\mathrm{C}$, 38.65; H, 2.34; F, 10.19; N, 17.53; P, 5.54\%. Found: C, 38.38; H, 2.11; F, 9.98; N, 17.35; P, 5.39\%. $\mathrm{M} / \mathrm{S}($ Int.\%): 555(M+3, 1.00), 234(11.11), 231(100), 136(18.2), 69(5.00).

6c: Deep brown solid, yield $88 \%$, m.p: $273^{\circ} \mathrm{C}-275^{\circ} \mathrm{C}$. IR spectrum $\bar{v}\left(\mathrm{~cm}^{-1}\right)$ : 3446 $(\mathrm{OH}), 3323(\mathrm{NH}), 3078($ aromatic $\mathrm{CH}), 2970$ (aliphatic $\mathrm{CH}), 1704(\mathrm{C}=\mathrm{O})$, 1615(C=N), 1555, 1307 (asym., sym. $\mathrm{NO}_{2}$ ), $1271(\mathrm{C}-\mathrm{F}), 1158(\mathrm{P}=\mathrm{O}), 1093(\mathrm{O}-\mathrm{P}-\mathrm{O})$, 970, 825(aromatic $\mathrm{CH}), 604(\mathrm{C}-\mathrm{F})$. Calculated $\mathrm{C}_{18} \mathrm{H}_{13} \mathrm{~F}_{3} \mathrm{~N}_{7} \mathrm{O}_{9} \mathrm{P}\left(\mathrm{M}^{+}\right.$559): C, 38.65; H, 2.34; F, 10.19; N, 17.53; P, 5.54\%. Found: C, 38.40; H, 2.13; F, 10.01; N, 17.40; $\mathrm{P}, 5.33 \%$.

6d: Deep brown solid, yield $78 \%$, m.p: $251^{\circ} \mathrm{C}-253^{\circ} \mathrm{C}$. IR spectrum $\bar{v}\left(\mathrm{~cm}^{-1}\right)$ : 3447 $(\mathrm{OH}), 3323(\mathrm{NH}), 3080$ (aromatic $\mathrm{CH}), 2970$ (aliphatic $\mathrm{CH}), 1702(\mathrm{C}=\mathrm{O})$, 1614(C=N), 1557, 1304(asym. \& sym. $\left.\mathrm{NO}_{2}\right), 1269(\mathrm{C}-\mathrm{F}), 1218(\mathrm{C}-\mathrm{P}=\mathrm{O}), 1060$ (P-O-H) 975, 900, 880, 790(aromatic CH), 729(C-Br), 600(C-F). Calculated $\mathrm{C}_{18} \mathrm{H}_{13} \mathrm{BrF}_{3} \mathrm{~N}_{6} \mathrm{O}_{7} \mathrm{P}\left(\mathrm{M}^{+}\right.$593): C, 36.45; H, 2.21; Br, 13.47; F, 9.61; N, 14.17; P, 5.22\%. Found: C, 36.15; H, 2.15; Br, 13.47; F, 9.39; N, 14.05; P, 5.11\%.

6e: Deep brown solid, yield $74 \%$, m.p: $263^{\circ} \mathrm{C}-265^{\circ} \mathrm{C}$. IR spectrum $\bar{v}\left(\mathrm{~cm}^{-1}\right)$ : 
3446 $(\mathrm{OH}), 3321(\mathrm{NH}), \quad 3080($ aromatic $\mathrm{CH}), 2970$ (aliphatic $\mathrm{CH}), \quad 1702(\mathrm{C}=\mathrm{O})$, 1615(C=N), 1557, 1307(asym. \& sym. $\left.\mathrm{NO}_{2}\right), 1269(\mathrm{C}-\mathrm{F}), 1219(\mathrm{C}-\mathrm{P}=\mathrm{O}), 1060$ (P-O-H) 980, 900, 870, (aromatic CH), 748(C-Cl), 605(C-F). Calculated $\mathrm{C}_{18} \mathrm{H}_{13} \mathrm{ClF}_{3} \mathrm{~N}_{6} \mathrm{O}_{7} \mathrm{P}\left(\mathrm{M}^{+}\right.$548): C, 39.40; H, 2.39; $\mathrm{Cl}, 6.46 ; \mathrm{F}, 10.39 ; \mathrm{N}, 15.32 ; \mathrm{P}$, 5.64\%. Found: C, 39.15; H, 2.19; Cl, 6.31; F, 10.15; N, 15.20; P, 5.45\%.

6f: Brown solid, yield $83 \%$, m.p: $245^{\circ} \mathrm{C}-247^{\circ} \mathrm{C}$. IR spectrum $\bar{v}\left(\mathrm{~cm}^{-1}\right)$ : 3448(OH), 3325(NH), 3088(NH), 1703(C=O), 1616(C=N), 1517, 1324(asym. \& sym. $\mathrm{NO}_{2}$ ), $1240(\mathrm{C}-\mathrm{F}), 1216(\mathrm{C}-\mathrm{P}=\mathrm{O}), 1060(\underline{\mathrm{P}-\mathrm{O}}-\mathrm{H})$ 988, 910, 880, 790(aromatic $\mathrm{CH}$ ), 607(C-F). ${ }^{1} \mathrm{H}$ NMR(400 MHz, DMSO-d $\left.{ }_{6}\right) \delta(\mathrm{ppm}): 8.35(\mathrm{~s}, 1 \mathrm{H}, \mathrm{CH}-\mathrm{N}), 8.04-7.48 \&$ 7.47 - 6.75(each d, d, $7 \mathrm{H}$, aromatic protons), 4.98(s, $1 \mathrm{H}, \mathrm{OH}), 4.95(\mathrm{~s}, 1 \mathrm{H}, \mathrm{OH})$, 4.011(s, $1 \mathrm{H}, \mathrm{OH}) .{ }^{13} \mathrm{C}$ NMR(100 MHz, DMSO-d $\left.{ }_{6}\right) \delta(\mathrm{ppm}): 173.44(\mathrm{C}=\mathrm{O}), 153$, 152(C-O), 145.81(C-F), 135.12(NCN), 129.28 - 126.47(aromatic carbons), $114.68,113.67\left(\mathrm{C}_{5}, \mathrm{C}_{6}\right.$ of 1,2,4-triazine). Calculated $\mathrm{C}_{18} \mathrm{H}_{13} \mathrm{~F}_{4} \mathrm{~N}_{6} \mathrm{O}_{7} \mathrm{P}\left(\mathrm{M}^{+}\right.$532): $\mathrm{C}$, 40.62; H, 2.46; F, 14.28; N, 15.79; P, 5.82\%. Found: C, 40.45; H, 2.30; F, 14.12; N, 15.56; P, 5.69\%. M/S(Int.\%): 534(M+2, 5.18), 234(43.1), 204(80.33), 136(22.15), 121(8.9), 96(12.15), 95(100).

\section{The Antioxidant Evaluation}

1,1-Diphenyl-2-picrylhydrazyl (DPPH) use to produce and reduce the odd electron stable-free radical which showed a strong UV-absorption maximum at $\lambda=$ $517 \mathrm{~nm}$. The new systems obtained dissolved in DMSO/EtOH at $150 \& 300$ $\mu \mathrm{mol} \cdot \mathrm{L}^{-1}$ added to DPPH at $100 \mu \mathrm{mol} \cdot \mathrm{L}^{-1}$. The tube kept at room temperature for 20 minutes and the absorption measured at $\lambda 517 \mathrm{~nm}$. The difference between the test and the control taken as the percent scavenging of the DPPH radical by use the formula: $\%$ inhibition $=(A B-A A) / A B \times 100$

where $A B$ : absorption of blank; $A A$ : absorption of the tested compound. The radical scavenging activity of ascorbic acid also measured and compared with that of the different synthesized compounds [27]. The observed data on the antioxidant-activated of the compounds and control shown in Table 1.

From the results obtained (Table 1 ) we can conclude that:

Presence of $\mathrm{CF}_{3}$ and $\mathrm{NO}_{2}$ of 6-aryl-1,2,4-triazinone and $\alpha$-amino phosphonate bearing 3-substituted amino-1,2,4-triazinones deployed a good to perfect scavenging activities. The ordering activity is $6 \mathrm{f}>6 \mathrm{e}>6 \mathrm{~d}>6 \mathrm{~b}>6 \mathrm{a}>6 \mathrm{c}$, which mainly attribute to the presence of C-halogen and $\mathrm{C}^{-\mathrm{NO}_{2}}$ of aryl-amino derivatives. The activity of $\alpha$-amino phosphonic acids 6 is higher than the activity of $\alpha$-amino phosphonates 5 . Also, high activity of $\mathbf{6 f}$ comparing with the other systems is may be due to a rich of fluorine atoms bonded to a 1,2,4-triazinone moiety, and the phosphonate groups are the potent antioxidant agent.

\section{Conclusion}

In the search for new antioxidant agents, the present work reports a simple route to synthetic novel fluorine substituted nitroaryl-1,2,4-triazine bearing $\alpha$-amino phosphonic acids. Presence of rich aliphatic/aromatic fluorine atoms and nitro 
Table 1. The DPPH radical scavenging activity of the novel fluorinated $\alpha$-amino phosphonates and the related $\alpha$-amino phosphonic acids at 150 and $300 \mu \mathrm{mol} \cdot \mathrm{L}^{-1}$.

\begin{tabular}{ccc}
\hline \multirow{2}{*}{ Compd. No. } & \multicolumn{2}{c}{ DPPH \% inhibition antioxidant \pm SD } \\
\cline { 2 - 3 } & $150 \mu \mathrm{mol} \cdot \mathrm{L}^{-1}$ & $300 \mu \mathrm{mol} \cdot \mathrm{L}^{-1}$ \\
\hline $\mathbf{5 a}$ & $52.08 \pm 0.05$ & $55.50 \pm 0.15$ \\
\hline $\mathbf{5 c}$ & $52.20 \pm 0.20$ & $55.65 \pm 0.29$ \\
$\mathbf{5 d}$ & $51.76 \pm 0.21$ & $53.39 \pm 0.11$ \\
$\mathbf{5 e}$ & $51.88 \pm 0.15$ & $51.78 \pm 0.08$ \\
5f & $56.01 \pm 0.14$ & $59.01 \pm 0.05$ \\
6a & $52.85 \pm 0.11$ & $60.00 \pm 0.01$ \\
6b & $56.65 \pm 0.25$ & $61.77 \pm 0.31$ \\
6c & $53.25 \pm 0.11$ & $73.44 \pm 0.05$ \\
6d & $53.25 \pm 0.01$ & $55.69 \pm 0.05$ \\
6e & $60.01 \pm 0.01$ & $64.66 \pm 0.05$ \\
6f & $61.33 \pm 0.13$ & $64.55 \pm 0.15$ \\
Ascorbic acid & $71.70 \pm 0.11$ & $64.55 \pm 0.15$ \\
\hline & 43.00 & 50.70 \\
\hline
\end{tabular}

groups bonded to 1,2,4-triazinone bearing $\alpha$-amino phosphonic acids enhance the antioxidant activities, which may use the feature of medicinal treatments.

\section{References}

[1] Abdel-Rahman, R.M., Ali, T.E. and Abdel-Kariem, S.M. (2016) Methods for Synthesis of $\mathrm{N}$-Hetrocyclyl/Hetroaryl- $\alpha$-Aminophosphonates and $\alpha$-(Azahetrocyclyl) Phosphonates. ARKIVOC, 1, 183-211.

[2] Gholivand, K., Shariatinia, Z., Mahzouni, H.R. and Amiri, S. (2007) Phosphorus Heterocycles: Synthesis, Spectroscopic Study and X-Ray Crystallography of Some New Diazaphosphorinanes. Structural Chemistry, 18, 653-660. https://doi.org/10.1007/s11224-007-9197-3

[3] Abdel-Rahman, R.M. and Ali, T.E. (2013) Synthesis and Biological Evaluation of Some New Polyfluorinated 4-Thiazolidinone and $\alpha$-Aminophosphonic Acid Derivatives. Monatsheftefür Chemie-Chemical Monthly, 144, 1243-1252. https://doi.org/10.1007/s00706-013-0934-6

[4] Peyman, A., Budt, K.H., Spanig, J., Stowasser, B. and Ruppert, D. (1992) $\mathrm{C}_{2}$-Symmetric Phosphinic Acid Inhibitors of HIV Protease. Tetrahedron Letters, 33, 4549-4552. https://doi.org/10.1016/S0040-4039(00)61309-6

[5] Markwell, R. (2000) Aminophosphonic and Aminophosphinic Acid Derivatives as Inhibitors of Human Collagenase. In: Kukhar, V.P. and Hudson, H.R., Eds., Aminophosphonic and Aminophosphinic Acids: Chemistry and Biological Activity, Wiley, Chichester, 579-621.

[6] Kenawy, E.-R.S., Azaam, M.M. and Saad-Allah, K.M. (2015) Synthesis and Antimicrobial Activity of $\alpha$-Aminophosphonates Containing Chitosan Moiety. Arabian Journal of Chemistry, 8, 427-432. https://doi.org/10.1016/j.arabjc.2013.12.029

[7] Reddy, Y.H., Kumar, B.S., Reddy, G.C., Dadapeer, E. and Reddy, K.S. (2012) Syn- 
thesis and Bioassay of $\alpha$-Aminophosphonates. Der Chemica Sinica, 3, 817-823.

[8] Zhang, Y., Bai, S., Song, B., Bhadury, P.S., Hu, D., Yang, S., Zhang, X., Fan, H. and Lu, P. (2010) Enantioseparation and Plant Virucidal Bioactivity of New Quinazoline Derivatives with $\alpha$-Aminophosphonate Moiety. Journal of Chromatography B, 878, 1285-1289. https://doi.org/10.1016/j.jchromb.2009.11.024

[9] Ali, T.E., Abdel-Aziz, S.A., Somaya, M., Mohamed, E.H.A. and Abdel-Kariem, S.M. (2013) Synthesis and Biological Evaluations of a Series of Novelazolyl, Azinyl and Azepinyl Phosphonates. Heterocycles, 87, 2513-2522. https://doi.org/10.3987/COM-13-12836

[10] Abdel-Rahman, R.M., Makki, M.S., Ali, T.E. and Ibrahim, M.A. (2015) 1,2,4-Triazine Chemistry Part IV: Synthesis and Chemical Behavior of 3-Functionalized 5,6-Diphenyl-1,2,4-Triazines towards Some Nucleophilic and Electrophilic Reagents. Journal of Heterocyclic Chemistry, 52, 1595-1607. https://doi.org/10.1002/jhet.2014

[11] Abdel-Rahman, R.M., Makki, M.S.T., Ali, T.E. and Ibrahim, M.A. (2013) 1,2, 4-Triazine Chemistry Part III: Synthetic Strategies to Functionalized Bridgehead Nitrogen Heteroannulated 1,2,4-Triazine Systems and Their Regiospecific and Pharmacological Properties. Current Organic Synthesis, 10, 136-160.

[12] Abdel-Rahman, R.M. and Saad, H.A. (2016) Synthesis and Chemical Behavior of 1,2,4-Triazine Derivatives Bearing Phosphorus Amides as Donor-Acceptors: A Review. Current Organic Synthesis, 13, 408-425. https://doi.org/10.2174/1570179412666150905001956

[13] Al-Romaizan, A.N., Makki, M.S.T. and Abdel-Rahman, R.M. (2014) Synthesis of New Fluorine/Phosphorus Substituted 6-(2'-Amino Phenyl)-3-Thioxo-1,2,4-Triazin-5(2H, 4H)One and Their Related Alkylated Systems as Molluscicidal Agent as against the Snails Responsible for Bilharziasis Diseases. International Journal of Organic Chemistry, 4, 154-168.

[14] Ali, T.E., Abdel-Rahman, R.M., Hanafy, F.I. and El-Edfawy, S.M. (2008) Synthesis and Molluscicidal Activity of Phosphorus-Containing Heterocyclic Compounds Derived from 5,6-Bis (4-Bromophenyl)-3-Hydrazino-1,2,4-Triazine. Phosphorus, Sulfur, and Silicon and the Related Elements, 183, 2565-2577. https://doi.org/10.1080/10426500801967864

[15] Abdel-Rahman, R.M., Ibrahim, M.A. and Ali, T.E. (2010) 1,2,4-Triazine Chemistry Part II: Synthetic Approaches for Phosphorus Containing 1,2,4-Triazine Derivatives. European Journal of Chemistry, 1, 388-396. https://doi.org/10.5155/eurjchem.1.4.388-396.154

[16] Ali, T.E. (2009) Synthesis and Antibacterial Activity of Some New Thiadiaza/Triazaphospholes, Thiadiaza/Triaza/Tetrazaphosphinines and Thiadiaza/Tetrazaphosphepines Containing 1,2,4-Triazinone Moiety. European Journal of Medicinal Chemistry, 44, 4539-4546. https://doi.org/10.1016/j.ejmech.2009.06.022

[17] Abdel-Rahman, R.M. (2002) Chemoselectiveheterocyclization of Pharmacological Activities of New Heterocycles-A Review: Synthesis of New Phosphaheterobicyclic Systems Containing 1,2,4-triazine Moiety Part IX: Straightforward Synthesis of New Fluorine Bearing 5-Phospha-1,2,4-triazin/1,2,4-triazepine-3 thiones. Trends in $\mathrm{He}$ terocyclic Chemistry, 8, 187-195.

[18] Makki, M.S.T., Abdel-Rahman, R.M. and AbuAli, O.A. (2015) Synthesis of New Fluorinated 1,2,4-Triazino [3,4-b] [1,3,4]thiadiazolones as Antiviral Probes-Part II-Reactivities of Fluorinated 3-Aminophenyl-1,2,4-triazinothiadiazolon. International Journal of Organic Chemistry, 5, 153-165. 
https://doi.org/10.4236/ijoc.2015.53017

[19] Makki, M.S.T., Abdel-Rahman, R.M. and Khan, K.A. (2014) Fluorine Substituted 1,2,4-triazinones as Potential anti-HIV-1 and CDK2 Inhibitors. Journal of Chemistry, 2014, Article ID: 430573.

[20] Abdel-Rahman, R.M., Makki, M.S.T. and Al-Romaizan, A.N. (2014) Synthesis of Novel Fluorine Substituted Isolated and Fused Heterobicyclic Nitrogen Systems Bearing 6-(2'-Phosphorylanilido)-1,2,4-Triazin-5-One Moiety as Potential Inhibitor towards HIV-1 Activity. International Journal of Organic Chemistry, 4, 247-268. https://doi.org/10.4236/ijoc.2014.44028

[21] Abdel-Rahman, R.M., Makki, M.S.T. and Bawazir, W.A. (2011) Synthesis of Some More Fluorine Heterocyclic Nitrogen Systems Derived From Sulfa Drugs as Photochemical Probe Agents for Inhibition of Vitiligo Disease-Part I. E-Journal of Chemistry, 8, 405-414. https://doi.org/10.1155/2011/586063

[22] Abdel-Rahman, R.M. (1991) Synthesis and Anti-Human Immune Virus Some New Fluorine-Containing Substituted-3-Thioxo-1,2,4-Triazin-5-Ones. IL Farmaco, 42, 379-389.

[23] Abdel-Rahman, R.M. (2001) Chemistry of Uncondensed 1,2,4-Triazines, Part IV. Synthesis and Chemistry of Bioactive 3-Amino-1,2,4-Triazines and Related Compounds-An Overview. Pharmazie, 56, 275-286.

[24] Ali, T.E. (2009) Synthesis of Some New 1,3,2-Oxazaphosphinine, 1,3,2-Diazaphosphinine, Acyclic, and/or Cyclic $\alpha$-Aminophosphonate Derivatives Containing the Chromone Moiety. Phosphorus, Sulfur, and Silicon and the Related Elements, 185, 88-96. https://doi.org/10.1080/10426500802713309

[25] Ali, T.E. and Halacheva, S.S. (2009) Synthetic Approach for Novel Bis( $\alpha$-aminophosphornic Acid) Derivatives of Chromone Containing 1,2,4,3-Triazaphosphole Moieties. Heteroatom Chemistry, 20, 117-122. https://doi.org/10.1002/hc.20520

[26] Pandeya, S.N., Kumar, R., Pathak, A.K. and Nath, G. (2010) Synthesis and Biological Evaluation of Triazine Derivatives. Der Pharma Chemica, 2, 257-266.

[27] Siddhuraju, P. and Becker, K. (2007) The Antioxidant and Free Radical Scavenging Activities of Processed Cowpea (Vignaunguiculata (L.) Walp.) Seed Extracts. Food Chemistry, 101, 10-19. https://doi.org/10.1016/j.foodchem.2006.01.004 